\title{
Parâmetros cinéticos da degradação in vitro de alimentos incubados com inóculo microbiano de diferentes espécies de ruminantes
}

[Kinetic parameters of the ruminal in vitro degradation of feedstuffs given to different ruminant species]

\author{
A.R.G.F. Bezerra ${ }^{1}$, P. Malafaia ${ }^{2}$, M.C. Mancini ${ }^{3}$, E.S. Bezerra ${ }^{4}$, R.A.M. Vieira ${ }^{5}$ \\ ${ }^{1}$ IBAMA - SCEN - Brasília, DF \\ ${ }^{2} \mathrm{IZ}$ - DNAP - Universidade Federal Rural do Rio de Janeiro \\ BR 465, km 7 \\ 23851-970 - Seropédica, RJ \\ ${ }^{3}$ IT - DTQ - UFRRJ - Seropédica, RJ \\ ${ }^{4} \mathrm{IZ}$ - DPA - UFRRJ - Seropédica, RJ \\ ${ }^{5}$ CCTA - LZNA - UENF - Campos dos Goytacases, RJ
}

\begin{abstract}
RESUMO
Parâmetros cinéticos da degradação ruminal de alguns alimentos utilizados para ruminantes de zoológicos foram estimados mediante incubação in vitro com líquido ruminal de audade (Ammotragus lervia), cervo sambar (Cervus unicolor), elande (Taurotragus oryx), bovino (Bos taurus), bubalino (Bubalus bubalis), caprino (Capra hircus) e ovino (Ovis aries). Os parâmetros cinéticos foram estimados pela técnica da produção de gás, cujos dados foram ajustados pelos modelos de um e de duplo compartimento. Não foram detectadas diferenças nos parâmetros cinéticos que permitissem agrupar os alimentos (fibrosos $\times$ não fibrosos) e os animais (domésticos $\times$ silvestres). $O$ modelo de duplo compartimento foi o mais adequado para a estimação dos parâmetros cinéticos da degradação ruminal. Inóculo microbiano oriundo de ruminantes domésticos não é recomendado para estimar parâmetros cinéticos da degradação ruminal de alimentos utilizados para ruminantes silvestres de zoológicos.
\end{abstract}

Palavras-chave: ruminante, alimento, valor nutritivo, degradação ruminal, zoológico

\begin{abstract}
The estimation of the ruminal kinetic parameters of pumpkin, potato-sweet, beet, broccoli, carrot, alfalfa hay, alfalfa pellet and bean, currently used for feeding wild and domestic ruminants raised in the Rio de Janeiro Zoo, was made through in vitro incubation of the feedstuffs together with ruminal fluid obtained from aoudad (Ammotragus lervia), sambar deer (Cervus unicolor), eland (Taurotragus oryx), cattle (Bos taurus), buffalo (Bubalus bubalis), goat (Capra hircus) and sheep (Ovis aries). The gas production technique was used to obtain gas profiles, and the data were fitted by the mono or double compartmental model. The kinetic parameters were discrepant among both, animals and feedstuffs, and the double compartmental model gave the best estimation. Ruminal inocula from domestic ruminants can not be used to estimate the kinetic parameters of ruminal degradation of feedstuffs for wild ruminants.
\end{abstract}

Keywords: ruminant, feedstuff, nutritive value, ruminal degradation, zoo animal

Recebido para publicação em 17 de março de 2004

Recebido para publicação, após modificações, em 2 de setembro de 2004

*Autor para correspondência (corresponding author)

E-mail: malafaia-ufrrj@yahoo.com.br 


\section{INTRODUÇÃO}

Muitas espécies de animais silvestres correm perigo de extinção, pois as áreas naturais protegidas compreendem aproximadamente $3 \%$ dos continentes (Sick, 1997). A destruição e a fragmentação de ecossistemas para dar lugar às monoculturas e à criação de animais domésticos são os principais empecilhos para a manutenção de populações viáveis de animais silvestres na natureza.

Os zoológicos assumiram a necessidade de conciliar sua atividade de exibição de animais ao público com as atividades de educação $\mathrm{e}$ pesquisa. Assim, adaptaram suas estruturas para atender a esses objetivos, oferecendo condições para que espécies raras e extremamente sensíveis ao estresse pudessem ser cuidadosamente observadas por meio de técnicas de manejo nutricional e reprodutivo.

O sistema intensivo de criação, utilizado pela maioria dos zoológicos brasileiros, é caracterizado pelo fornecimento de vários tipos de alimentos diretamente no comedouro, impondo a necessidade de avaliar nutricionalmente os produtos oferecidos. Essa avaliação é necessária devido à pouca informação a respeito das exigências nutricionais dos animais silvestres, tornando-os, em sua grande maioria, animais de baixa eficiência reprodutiva e de difícil manutenção.

$\mathrm{Na}$ área de alimentação e nutrição de animais silvestres, o número de pesquisas tem aumentado substancialmente, porém a maioria dos estudos se desenvolve na natureza, avaliando a preferência dos animais por determinados alimentos e identificando os mais freqüentemente consumidos. Essa abordagem é importante no sentido de oferecer subsídios para se conhecer mais sobre os hábitos alimentares dos animais. Entretanto, pela diversidade de itens alimentares encontrados pelas espécies na natureza, ao longo do ano, fica difícil balancear nutricionalmente determinadas dietas em cativeiro.

Para estudos na área de nutrição, a busca por métodos menos invasivos é de extrema importância, uma vez que, tratando-se de animais silvestres, que vivem constantemente sob condições de estresse, qualquer contenção mal conduzida pode levar à morte do animal, resultando em perda econômica.

O objetivo deste trabalho foi estimar e comparar os parâmetros cinéticos da degradação ruminal de diferentes alimentos incubados com inóculo microbiano de várias espécies de ruminantes.

\section{MATERIAL E MÉTODOS}

Os procedimentos de coleta do líquido ruminal dos animais silvestres foram realizados na Fundação Jardim Zoológico da cidade do Rio de Janeiro (Fundação RIOZOO), e os inóculos dos animais domésticos foram coletados em animais da Universidade Federal Rural do Rio de Janeiro. Os alimentos estudados foram batata-doce (Ipomoea batatas), vagem (Phaseolus vulgaris), abóbora (Cucurbita moschata), brócolis (Brassica oleracea), cenoura (Daucus carota), beterraba (Beta vulgaris var. esculenta) e alfafa (Medicago sativa) nas formas peletizada e fenada.

Durante três dias consecutivos, por ocasião do arraçoamento dos animais, foram colhidas amostras compostas dos ingredientes que compunham suas dietas. As amostras foram présecas a $55^{\circ} \mathrm{C}$ durante 72 horas e posteriormente moídas em moinho dotado com peneira de $1,0 \mathrm{~mm}$. Foram realizadas análises de matéria seca (MS), cinzas, extrato etéreo (EE) e proteína bruta (PB), seguindo os procedimentos sugeridos pela AOAC (Official..., 1990). A metodologia empregada nas análises da fibra em detergente neutro (FDN) e da fibra em detergente ácido (FDA) foram as propostas por Van Soest et al. (1991). Os carboidratos totais (CHT) foram calculados como $\mathrm{CHT}(\mathrm{g})=100$ - $(\mathrm{PB}+\mathrm{MM}+\mathrm{EE})$, e a fração de carboidratos não estruturais (CNE) foi obtida pela diferença entre o teor de carboidratos totais (CHT) e o teor de fibra em detergente neutro corrigida para cinzas e proteínas (FDNcp). A composição bromatológica dos alimentos é apresentada na Tab. 1. 
Tabela 1. Composição bromatológica de alimentos utilizados em zoológicos

\begin{tabular}{lcccccccccc}
\hline Alimento & MS $^{1}$ & Cinzas $^{1}$ & MO $^{1}$ & PB $^{1}$ & EE $^{1}$ & CHT $^{1}$ & CNE $^{1}$ & Fibra $^{1}$ & Lignina $^{2}$ & Celulose $^{2}$ \\
\hline Abóbora & 70,71 & 4,33 & 66,37 & 7,62 & 1,53 & 57,23 & 42,63 & 14,59 & 40 & 132 \\
Batata doce & 290,91 & 7,31 & 283,59 & 9,35 & 4,51 & 269,74 & 210,42 & 59,32 & 25 & 21 \\
Beterraba & 91,95 & 9,30 & 82,60 & 13,07 & 0,80 & 68,77 & 53,16 & 15,60 & 28 & 75 \\
Brócolis & 88,37 & 11,06 & 77,34 & 29,28 & 4,88 & 43,15 & 24,46 & 18,69 & 65 & 115 \\
Cenoura & 94,21 & 0,72 & 93,48 & 5,48 & 1,76 & 86,26 & 73,76 & 12,49 & 65 & 75 \\
Feno de alfafa & 883,60 & 114,49 & 769,11 & 173,44 & 24,34 & 571,33 & 83,39 & 487,9 & 150 & 243 \\
Alfafa peletizada & 883,89 & 90,76 & 793,14 & 150,34 & 15,64 & 627,15 & 49,76 & 577,4 & 121 & 287 \\
Vagem & 75,74 & 7,56 & 68,14 & 24,37 & 0,90 & 42,90 & 4,20 & 38,70 & 132 & 222 \\
\hline
\end{tabular}

${ }^{1} \mathrm{Em} \mathrm{g} \mathrm{kg}{ }^{-1}$ de matéria natural. ${ }^{2} \mathrm{Em} \mathrm{g} \mathrm{kg}^{-1}$ de fibra.

$\mathrm{MS}=$ matéria seca; $\mathrm{MO}=$ matéria orgânica; $\mathrm{PB}=$ proteína bruta; $\mathrm{EE}=$ extrato etéreo; $\mathrm{CHT}=$ carboidratos totais; $\mathrm{CNE}=$ carboidratos não estruturais.

O inóculo microbiano foi retirado do ambiente ruminal de apenas um animal das seguintes espécies: búfalo (Bubalus bubalis), bovino (Bos taurus), cabra doméstica (Capra hircus), carneiro (Ovis aries), cervo sambar (Cervus unicolor), audade (Ammotragus lervia) e elande (Taurotragus oryx). Para a retirada do inóculo, foi utilizada uma sonda gástrica acoplada a uma bomba odontológica de sucção a vácuo. A contenção dos animais domésticos foi feita sem o uso de sedativos, enquanto a dos animais silvestres foi conduzida quimicamente, à base de cloridrato de xilazina e cloridrato de ketamina, na proporção de $1 \mathrm{mg} / \mathrm{kgPV}$ de xilazina + $10 \mathrm{mg} / \mathrm{kgPV}$ de ketamina para o audade, $3 \mathrm{mg} / \mathrm{kg}$ de xilazina $+15 \mathrm{mg} / \mathrm{kg}$ de ketamina para o elande e $3 \mathrm{mg} / \mathrm{kg}$ de xilazina $+10 \mathrm{mg} / \mathrm{kg}$ de ketamina para o cervo sambar. Imediatamente após a retirada, o inóculo foi filtrado sob ventilação com $\mathrm{CO}_{2}$, acondicionado em garrafa térmica a $39^{\circ} \mathrm{C}$ e levado para o laboratório.

A produção cumulativa de gás foi obtida por incubações in vitro, empregando-se a metodologia descrita por Malafaia et al. (1999). $\mathrm{O}$ volume dos gases foi medido à 1 e às $2,3,4$, $6,9,12,24,36,48$ e 60 horas após a adição do inóculo ruminal.

Os dados da produção cumulativa dos gases foram analisados pelos modelos de um compartimento e de duplo compartimento (Schofield et al., 1994). O modelo de um compartimento foi representado pela expressão matemática:

$\mathrm{V}_{(\mathrm{t})}=\frac{\mathrm{V}_{\mathrm{fl}}}{\left[1+\mathrm{e}^{(2+4 \mathrm{c}(\mathrm{L}-\mathrm{T}))}\right]}$, em que

$c$ representa a taxa de degradação do alimento; $V_{(t)}$, o volume final de gás; $L$, a duração do evento de degradação inicial do alimento; e $T$, o intervalo de tempo de leitura do volume de gás.

O modelo de duplo compartimento foi representado por:

$\mathrm{V}_{(\mathrm{t})}=\frac{\mathrm{V}_{\mathrm{fl}}}{\left[1+\mathrm{e}^{\left(2+4 \mathrm{~m}_{1}(\mathrm{~L}-\mathrm{T})\right)}\right]}+\frac{\mathrm{V}_{\mathrm{f} 2}}{\left.1+\mathrm{e}^{\left(2+4 \mathrm{~m}_{2}(\mathrm{~L}-\mathrm{T})\right)}\right]}$, em que

$V_{f 1}$ representa o volume máximo de gás para a fração de rápida digestão; $V_{f 2}$ representa $\mathrm{o}$ volume máximo de gás para a fração de lenta digestão; $m_{1}$ equivale à taxa de crescimento específico para a fração de rápida degradação; $m_{2}$ equivale à taxa de crescimento específico para a fração de lenta degradação; e $L$ representa a duração dos eventos iniciais (fase de latência) de digestão, comum às duas fases.

Para a realização dos ajustes, utilizou-se o procedimento não linear do SAEG (Sistema..., 1995). Os dados foram analisados quanto à normalidade e à homogeneidade do erro experimental, utilizando-se o teste de Cochran, mediante os procedimentos disponíveis no SAEG (Sistema..., 1995).

Para a estimativa do rendimento microbiano no rúmen (gcélulas/gcarboidrato fermentado), foram empregadas as equações utilizadas por Russell et al. (1992):

$\mathrm{Y}_{\mathrm{cnf}}=\frac{0,4 \cdot \mathrm{m}_{1}}{0,4 \cdot 0,15+\mathrm{m}_{1}} \mathrm{e}_{\mathrm{Y}_{\mathrm{cf}}}=\frac{0,4 \cdot \mathrm{m}_{2}}{0,4 \cdot 0,05+\mathrm{m}_{2}}$, em que

$Y_{c n f}$ equivale ao rendimento microbiano da população não fibrolítica; e $Y_{c f}$, ao da população fibrolítica; 0,4 significa o rendimento teórico máximo dos microrganismos ruminais; 0,05 equivale à exigência de mantença das bactérias 
que utilizam carboidratos fibrosos; e 0,15 , à exigência de mantença das bactérias que utilizam carboidratos não fibrosos.

Para a análise de variância, empregou-se o modelo:

$Y_{e i j}=\mu+E_{i}+A_{j}+E_{i j}+e_{i j k}$, em que

$Y_{i j k}$ equivale à k-ésima estimativa do parâmetro cinético $\left(L, m_{1}\right.$ e $\left.m_{2}\right)$, obtida após o ajuste do modelo ao k-ésimo perfil de produção de gases gerado pela incubação do j-ésimo alimento, com o inóculo extraído do rúmen da i-ésima espécie; $\mu$ é uma constante inerente ao modelo; $E_{\mathrm{i}}$ representa a i-ésima espécie; $A_{\mathrm{j}}$ corresponde ao j- ésimo alimento; e $e_{i j k}$ representa o erro aleatório, suposto normal e independentemente distribuído, com média zero e variância $\sigma^{2}$. As médias foram comparadas pelo teste Tukey $(\propto=5 \%)$, do programa SAEG (Sistema..., 1995).

\section{RESULTADOS E DISCUSSÃO}

Com exceção do brócolis, incubado com o inóculo do cervo sambar, a estimativa dos parâmetros do modelo de um compartimento resultou em níveis de confiança sempre inferiores aos obtidos com o modelo de duplo compartimento (Tab. 2).

Tabela 2. Tempo de latência $(L)$, taxa de degradação $(c)$, nível de confiança (NC) e coeficiente de determinação $\left(\mathrm{R}^{2}\right)$ de alimentos usados para várias espécies de ruminantes, estimados pelo modelo de um compartimento

\begin{tabular}{|c|c|c|c|c|c|c|c|c|}
\hline \multirow{2}{*}{ Alimento } & \multirow{2}{*}{ Parâmetro } & \multicolumn{7}{|c|}{ Espécie } \\
\hline & & Boi & Búfalo & Cabra & Ovelha & Audade & Cervo & Elande \\
\hline \multirow{4}{*}{ Abóbora } & $L(\mathrm{~h})$ & 2.83 & -1.48 & 1.78 & 0.92 & -0.24 & 5.48 & 3.49 \\
\hline & $c\left(\mathrm{~h}^{-1}\right)$ & 0.06 & 0.04 & 0.07 & 0.05 & 0.05 & 0.04 & 0.05 \\
\hline & $\mathrm{R}^{2}$ & 0.98 & 0.97 & 0.97 & 0.98 & 0.98 & 0.99 & 0.98 \\
\hline & $\mathrm{NC}(\%)$ & 96.03 & 94.32 & 94.61 & 97.82 & 97.60 & 98.53 & 97.74 \\
\hline \multirow{4}{*}{ Batata-doce } & $L(\mathrm{~h})$ & 3.48 & 0.75 & 2.83 & 2.48 & 1.97 & 5.04 & 5.12 \\
\hline & $c\left(\mathrm{~h}^{-1}\right)$ & 0.03 & 0.03 & 0.03 & 0.04 & 0.03 & 0.04 & 0.05 \\
\hline & $\mathrm{R}^{2}$ & 0.98 & 0.98 & 0.97 & 0.99 & 0.98 & 0.99 & 0.99 \\
\hline & $\mathrm{NC}(\%)$ & 96.15 & 96.34 & 95.99 & 98.11 & 97.87 & 98.96 & 99.06 \\
\hline \multirow{4}{*}{ Beterraba } & $L(\mathrm{~h})$ & 2.37 & -0.60 & 2.32 & 1.41 & -0.24 & 2.22 & 3.49 \\
\hline & $c\left(\mathrm{~h}^{-1}\right)$ & 0.09 & 0.05 & 0.08 & 0.06 & 0.06 & 0.04 & 0.05 \\
\hline & $\mathrm{R}^{2}$ & 0.98 & 0.97 & 0.98 & 0.99 & 0.98 & 0.98 & 0.98 \\
\hline & $\mathrm{NC}(\%)$ & 97.81 & 95.13 & 97.34 & 98.28 & 97.76 & 97.83 & 97.74 \\
\hline \multirow{4}{*}{ Brócolis } & $L(\mathrm{~h})$ & 3.53 & 0.44 & 3.04 & 2.09 & 3.59 & 4.76 & 4.11 \\
\hline & $c\left(\mathrm{~h}^{-1}\right)$ & 0.09 & 0.05 & 0.07 & 0.06 & 0.06 & 0.04 & 0.02 \\
\hline & $\mathrm{R}^{2}$ & 0.99 & 0.98 & 0.98 & 0.99 & 0.99 & 0.99 & 0.98 \\
\hline & $\mathrm{NC}(\%)$ & 98.46 & 96.34 & 97.57 & 98.77 & 98.57 & 98.42 & 96.49 \\
\hline \multirow{4}{*}{ Cenoura } & $L(\mathrm{~h})$ & 3.13 & -1.11 & 1.51 & 0.99 & 0.25 & 1.15 & 2.66 \\
\hline & $c\left(\mathrm{~h}^{-1}\right)$ & 0.07 & 0.04 & 0.06 & 0.06 & 0.05 & 0.04 & 0.05 \\
\hline & $\mathrm{R}^{2}$ & 0.98 & 0.97 & 0.98 & 0.98 & 0.98 & 0.98 & 0.99 \\
\hline & $\mathrm{NC}(\%)$ & 97.02 & 94.76 & 96.07 & 97.52 & 96.73 & 96.97 & 98.28 \\
\hline \multirow{4}{*}{ Feno de alfafa } & $L(\mathrm{~h})$ & 4.60 & 0.31 & 3.03 & 5.01 & 3.71 & 12.31 & 4.63 \\
\hline & $c\left(\mathrm{~h}^{-1}\right)$ & 0.07 & 0.03 & 0.05 & 0.04 & 0.03 & 0.05 & 0.02 \\
\hline & $\mathrm{R}^{2}$ & 0.98 & 0.98 & 0.97 & 0.99 & 0.98 & 0.99 & 0.98 \\
\hline & $\mathrm{NC}(\%)$ & 96.78 & 96.53 & 95.58 & 98.25 & 97.57 & 98.82 & 96.47 \\
\hline \multirow{4}{*}{$\begin{array}{l}\text { Alfafa } \\
\text { peletizada }\end{array}$} & $L(\mathrm{~h})$ & 2.83 & 4.61 & 2.25 & 4.19 & 4.23 & 10.97 & 1.75 \\
\hline & $c\left(\mathrm{~h}^{-1}\right)$ & 0.08 & 0.04 & 0.07 & 0.04 & 0.04 & 0.05 & 0.03 \\
\hline & $\mathrm{R}^{2}$ & 0.98 & 0.99 & 0.97 & 0.99 & 0.99 & 0.99 & 0.98 \\
\hline & $\mathrm{NC}(\%)$ & 97.64 & 97.81 & 94.87 & 98.30 & 98.24 & 99.05 & 96.84 \\
\hline \multirow{4}{*}{ Vagem } & $L(\mathrm{~h})$ & 2.80 & 2.35 & 1.83 & 3.86 & 4.17 & 7.25 & 5.32 \\
\hline & $c\left(\mathrm{~h}^{-1}\right)$ & 0.08 & 0.05 & 0.06 & 0.04 & 0.04 & 0.05 & 0.02 \\
\hline & $\mathrm{R}^{2}$ & 0.99 & 0.99 & 0.98 & 0.99 & 0.99 & 0.99 & 0.98 \\
\hline & $\mathrm{NC}(\%)$ & 98.17 & 98.54 & 97.51 & 98.85 & 99.17 & 99.17 & 96.83 \\
\hline
\end{tabular}


$\mathrm{Na}$ medida em que os níveis de confiança observados para o modelo de um compartimento foram inferiores aos estimados para o modelo de duplo compartimento, o primeiro modelo deve ser preterido em favor desse último. De fato, nem todos os componentes alimentares são digeridos similarmente, e essas diferentes taxas de digestão, para as distintas frações alimentares, precisam ser identificadas de acordo com suas características cinéticas; justificando, assim, o melhor ajustamento do modelo de duplo compartimento para a estimação desses parâmetros.

A adequabilidade de um modelo pode ser avaliada pelo coeficiente de determinação $\left(\mathrm{R}^{2}\right)$. De acordo com Sampaio (1998), com $\mathrm{R}^{2}$ alto, o modelo fará melhores estimativas e será adequado aos dados obtidos. Por outro lado, $\mathrm{R}^{2}$ baixo não permite estimativas confiáveis, quer pela alta variabilidade da resposta medida, quer pelo fato de o modelo testado não ser adequado à dispersão dos dados observados.

Com exceção do brócolis incubado com o líquido ruminal do cervo e do elande, os coeficientes de determinação encontrados para o modelo de duplo compartimento foram sempre iguais ou superiores a 0,99 (Tab. 3), o que justifica a sua aplicação. Ao se utilizar o modelo de um compartimento, foi obtida grande amplitude de variação para os valores de $\mathrm{R}^{2} \mathrm{e}$, em boa parte das análises, eles foram inferiores a 0,99 (Tab. 2), confirmando que os dados não devem ser ajustados a esse modelo.

Com relação à duração do evento de degradação inicial do alimento (fase de latência), os valores negativos encontrados nos alimentos incubados com líquido ruminal do búfalo e do audade (Tab. 2) são estimativas espúrias do modelo de um compartimento, uma vez que, biologicamente, não haveria explicação para esse fenômeno. Entretanto, deve ser ressaltado que em sistemas in vitro, como o usado neste estudo, os valores de latência podem ser induzidos artificialmente em razão da incapacidade de se manter a perfeita anaerobiose do meio, da variação na pressão osmótica e da ausência de co-fatores nesse ambiente (Van Soest, 1994). Quando Van Milgen et al. (1991) propuseram um modelo de digestão, descrevendo-o como um processo de compartimentos seqüenciais, levaram em consideração a necessidade de incorporar $o$ fenômeno da latência, que se ajustava melhor a um modelo multicompartimental, fato plenamente ratificado pelos resultados aqui encontrados.

As taxas de degradação $m_{l}$ e $m_{2}$ não seguiram um padrão para todos os alimentos (Tab. 3). A tentativa de associar essas taxas com o teor de carboidratos fibrosos e de carboidratos não fibrosos foi inadequada, visto que, tanto nos alimentos com níveis elevados desses nutrientes, quanto nos de baixa concentração, as estimativas resultaram em taxas bastante discrepantes. Os efeitos do animal doador de inóculo não foram consistentes sobre as taxas de degradação da fração de carboidratos não fibrosos.

A incubação da abóbora, batata-doce, beterraba, brócolis e cenoura com o inóculo do cervo sambar resultou nas maiores taxas de degradação da fração de carboidratos fibrosos $\left(m_{2}\right)$ desses alimentos, que são ricos em carboidratos de reserva. Entretanto, o mesmo não se verificou com relação aos alimentos com maior conteúdo em carboidratos fibrosos. Para os demais animais, houve variações consideráveis nas estimativas dos parâmetros e não foram verificadas tendências em relação ao tipo de alimento em que a massa microbiana foi incubada. Nos alimentos ricos em carboidratos fibrosos (alfafa fenada ou peletizada), os inóculos do bovino e do caprino resultaram em maiores taxas de degradação da fração de carboidratos fibrosos $\left(m_{2}\right)$, quando comparadas às das demais espécies.

Há poucos estudos comparando o mecanismo de ação da microbiota entre espécies animais. $\mathrm{Na}$ natureza, o ruminante silvestre tende a se adaptar ao ambiente que o cerca, colocando-se à mercê da forragem predominante nesse ambiente. A seletividade, neste caso, poderia ter considerável influência sobre a taxa de digestão. É possível supor que, ao se coletar o inóculo de diferentes ruminantes, em situações de vida distintas, indiretamente se estaria promovendo "fermentação seletiva", que poderia levar a uma preferência da microbiota ali presente por determinado substrato em detrimento de outros. 
Tabela 3. Tempo de latência $(L)$, taxas de degradação $m_{l}$ e $m_{2}$, nível de confiança (NC) e coeficiente de determinação $\left(\mathrm{R}^{2}\right)$ de alimentos usados para várias espécies de ruminantes, estimados pelo modelo de duplo compartimento

\begin{tabular}{|c|c|c|c|c|c|c|c|c|}
\hline \multirow{2}{*}{ Alimento } & \multirow{2}{*}{ Parâmetro } & \multicolumn{7}{|c|}{ Espécie } \\
\hline & & Boi & Búfalo & Cabra & Ovelha & Audade & Cervo & Elande \\
\hline \multirow{5}{*}{ Abóbora } & $L(\mathrm{~h})$ & $4.58 \mathrm{ab}$ & $1.29 \mathrm{c}$ & $3.63 b$ & $0.86 \mathrm{c}$ & $0.40 \mathrm{c}$ & $6.05 \mathrm{a}$ & $3.45 b$ \\
\hline & $m_{l}\left(\mathrm{~h}^{-1}\right)$ & $0.226 \mathrm{ab}$ & $0.193 \mathrm{abc}$ & $0.350 \mathrm{a}$ & $0.072 \mathrm{~cd}$ & $0.073 \mathrm{~cd}$ & $0.038 \mathrm{~d}$ & $0.126 \mathrm{bcd}$ \\
\hline & $m_{2}\left(\mathrm{~h}^{-1}\right)$ & $0.028 b$ & $0.025 b$ & $0.027 b$ & $0.025 b$ & $0.026 \mathrm{~b}$ & $0.038 \mathrm{a}$ & $0.036 \mathrm{a}$ \\
\hline & $\mathrm{R}^{2}$ & 0.99 & 0.99 & 0.99 & 0.99 & 0.99 & 0.99 & 0.99 \\
\hline & $\mathrm{NC}(\%)$ & 99.83 & 99.84 & 99.93 & 99.43 & 99.59 & 99.67 & 99.70 \\
\hline \multirow{5}{*}{ Batata-doce } & $L(\mathrm{~h})$ & $5.75 \mathrm{ab}$ & $3.12 \mathrm{c}$ & $1.29 \mathrm{~d}$ & $3.71 b c$ & $4.35 \mathrm{abc}$ & $3.90 \mathrm{bc}$ & $6.17 \mathrm{a}$ \\
\hline & $m_{l}\left(\mathrm{~h}^{-1}\right)$ & $0.271 \mathrm{a}$ & $0.158 \mathrm{ab}$ & $0.111 b c$ & $0.112 b c$ & $0.126 a b c$ & $0.038 \mathrm{c}$ & $0.135 \mathrm{abc}$ \\
\hline & $m_{2}\left(\mathrm{~h}^{-1}\right)$ & $0.024 \mathrm{bc}$ & $0.019 \mathrm{c}$ & $0.022 \mathrm{c}$ & $0.029 b$ & $0.023 b c$ & $0.038 \mathrm{a}$ & $0.036 \mathrm{a}$ \\
\hline & $\mathrm{R}^{2}$ & 0.99 & 0.99 & 0.99 & 0.99 & 0.99 & 0.99 & 0.99 \\
\hline & $\mathrm{NC}(\%)$ & 99.70 & 99.70 & 99.99 & 99.75 & 99.79 & 99.23 & 99.80 \\
\hline \multirow{5}{*}{ Beterraba } & $L(\mathrm{~h})$ & $2.85 \mathrm{ab}$ & $1.30 \mathrm{c}$ & $3.61 \mathrm{a}$ & $1.34 b c$ & $0.81 \mathrm{c}$ & $3.01 \mathrm{a}$ & $4.01 \mathrm{a}$ \\
\hline & $m_{l}\left(\mathrm{~h}^{-1}\right)$ & $0.189 b c$ & $0.193 b c$ & $0.318 \mathrm{ab}$ & $0.084 \mathrm{c}$ & $0.253 \mathrm{ab}$ & $0.422 \mathrm{a}$ & $0.155 \mathrm{bc}$ \\
\hline & $m_{2}\left(\mathrm{~h}^{-1}\right)$ & $0.041 \mathrm{ab}$ & $0.030 \mathrm{c}$ & $0.041 \mathrm{ab}$ & $0.030 \mathrm{c}$ & $0.048 \mathrm{a}$ & $0.038 \mathrm{~b}$ & $0.027 \mathrm{c}$ \\
\hline & $\mathrm{R}^{2}$ & 0.99 & 0.99 & 0.99 & 0.99 & 0.99 & 0.99 & 0.99 \\
\hline & $\mathrm{NC}(\%)$ & 99.76 & 99.82 & 99.94 & 99.62 & 99.55 & 99.44 & 99.56 \\
\hline \multirow{5}{*}{ Brócolis } & $L(\mathrm{~h})$ & $4.26 \mathrm{a}$ & $1.16 \mathrm{c}$ & $4.32 \mathrm{a}$ & $2.14 b c$ & $4.12 \mathrm{a}$ & $3.21 \mathrm{ab}$ & $3.52 \mathrm{ab}$ \\
\hline & $m_{l}\left(\mathrm{~h}^{-1}\right)$ & $0.183 b$ & $0.156 \mathrm{~b}$ & $0.479 \mathrm{a}$ & $0.075 \mathrm{bc}$ & $0.063 \mathrm{bc}$ & $0.038 \mathrm{c}$ & $0.021 \mathrm{c}$ \\
\hline & $m_{2}\left(\mathrm{~h}^{-1}\right)$ & $0.039 \mathrm{a}$ & $0.027 b$ & $0.044 \mathrm{a}$ & $0.030 \mathrm{~b}$ & $0.027 \mathrm{bc}$ & $0.038 \mathrm{a}$ & $0.020 \mathrm{c}$ \\
\hline & $\mathrm{R}^{2}$ & 0.99 & 0.99 & 0.99 & 0.99 & 0.99 & 0.99 & 0.99 \\
\hline & $\mathrm{NC}(\%)$ & 99.96 & 99.84 & 99.92 & 99.64 & 99.48 & 98.13 & 98.73 \\
\hline \multirow{5}{*}{ Cenoura } & $L(\mathrm{~h})$ & $4.20 \mathrm{a}$ & $1.98 b c$ & $3.33 \mathrm{ab}$ & $0.90 \mathrm{c}$ & $0.93 \mathrm{c}$ & $2.59 \mathrm{ab}$ & $3.12 \mathrm{ab}$ \\
\hline & $m_{l}\left(\mathrm{~h}^{-1}\right)$ & $0.175 b c$ & $0.219 \mathrm{abc}$ & $0.245 \mathrm{ab}$ & $0.092 \mathrm{c}$ & $0.228 \mathrm{abc}$ & $0.380 \mathrm{a}$ & $0.148 b c$ \\
\hline & $m_{2}\left(\mathrm{~h}^{-1}\right)$ & $0.030 \mathrm{bcd}$ & $0.025 \mathrm{~d}$ & $0.030 \mathrm{~cd}$ & $0.031 \mathrm{bcd}$ & $0.039 \mathrm{a}$ & $0.037 \mathrm{ab}$ & $0.036 \mathrm{abc}$ \\
\hline & $\mathrm{R}^{2}$ & 0.99 & 0.99 & 0.99 & 0.99 & 0.99 & 0.99 & 0.99 \\
\hline & $\mathrm{NC}(\%)$ & 99.83 & 99.94 & 99.93 & 99.27 & 99.47 & 99.42 & 99.76 \\
\hline \multirow{5}{*}{ Feno de alfafa } & $L(\mathrm{~h})$ & $1.97 \mathrm{~b}$ & $2.29 \mathrm{~b}$ & $1.38 \mathrm{~b}$ & $1.91 \mathrm{~b}$ & $4.96 a$ & $2.29 \mathrm{~b}$ & $2.95 b$ \\
\hline & $m_{l}\left(\mathrm{~h}^{-1}\right)$ & $0.268 \mathrm{a}$ & $0.016 b$ & $0.461 \mathrm{a}$ & $0.302 \mathrm{a}$ & $0.048 b$ & $0.399 \mathrm{a}$ & $0.493 \mathrm{a}$ \\
\hline & $m_{2}\left(\mathrm{~h}^{-1}\right)$ & $0.033 \mathrm{a}$ & $0.016 \mathrm{c}$ & $0.030 \mathrm{ab}$ & $0.024 b$ & $0.009 \mathrm{~d}$ & $0.024 \mathrm{~b}$ & $0.008 \mathrm{~d}$ \\
\hline & $\mathrm{R}^{2}$ & 0.99 & 0.99 & 0.99 & 0.99 & 0.99 & 0.99 & 0.99 \\
\hline & $\mathrm{NC}(\%)$ & 99.84 & 99.76 & 99.89 & 99.78 & 99.97 & 99.99 & 99.06 \\
\hline \multirow{5}{*}{ Alfafa peletizada } & $L(\mathrm{~h})$ & $1.01 \mathrm{~d}$ & $3.93 \mathrm{a}$ & $0.98 \mathrm{~d}$ & $1.23 \mathrm{~cd}$ & $1.64 \mathrm{bcd}$ & $2.70 \mathrm{ab}$ & $3.03 \mathrm{abc}$ \\
\hline & $m_{l}\left(\mathrm{~h}^{-1}\right)$ & $0.260 \mathrm{ab}$ & $0.040 \mathrm{~cd}$ & $0.256 \mathrm{ab}$ & $0.443 \mathrm{a}$ & $0.036 \mathrm{~d}$ & $0.164 b c$ & $0.408 \mathrm{a}$ \\
\hline & $m_{2}\left(\mathrm{~h}^{-1}\right)$ & $0.034 \mathrm{a}$ & $0.016 \mathrm{~d}$ & $0.029 \mathrm{ab}$ & $0.028 \mathrm{ab}$ & $0.019 \mathrm{~cd}$ & $0.023 b c$ & $0.008 \mathrm{e}$ \\
\hline & $\mathrm{R}^{2}$ & 0.99 & 0.99 & 0.99 & 0.99 & 0.99 & 0.99 & 0.99 \\
\hline & $\mathrm{NC}(\%)$ & 99.78 & 99.92 & 99.94 & 99.96 & 99.19 & 99.99 & 99.07 \\
\hline \multirow{5}{*}{ Vagem } & $L(\mathrm{~h})$ & $0.85 b c$ & $2.05 \mathrm{~b}$ & $0.26 \mathrm{c}$ & $0.89 b c$ & $2.00 \mathrm{~b}$ & $1.64 b$ & $13.14 \mathrm{a}$ \\
\hline & $m_{l}\left(\mathrm{~h}^{-1}\right)$ & $0.152 \mathrm{ab}$ & $0.052 \mathrm{bcd}$ & $0.161 \mathrm{ab}$ & $0.298 \mathrm{a}$ & $0.029 \mathrm{~cd}$ & $0.120 \mathrm{abc}$ & $0.020 \mathrm{~d}$ \\
\hline & $m_{2}\left(\mathrm{~h}^{-1}\right)$ & $0.035 \mathrm{ab}$ & $0.028 \mathrm{c}$ & $0.036 \mathrm{a}$ & $0.029 b c$ & $0.004 \mathrm{~d}$ & $0.032 \mathrm{abc}$ & $0.009 \mathrm{~d}$ \\
\hline & $R^{2}$ & 0.99 & 0.99 & 0.99 & 0.99 & 0.99 & 0.99 & 0.99 \\
\hline & $\mathrm{NC}(\%)$ & 99.88 & 99.89 & 99.94 & 99.63 & 99.58 & 99.23 & 99.70 \\
\hline
\end{tabular}

Médias na linha seguidas de letras distintas diferem entre si $(\mathrm{P}<0,05)$

Foram detectadas diferenças $(\mathrm{P}<0,05)$ entre as fases de latência para os alimentos, destacandose sua maior duração para os inóculos que utilizaram batata-doce como substrato, enquanto que aqueles contendo alfafa peletizada apresentaram menor tempo de latência (Tab. 4).
Sendo a batata-doce, o brócolis, a vagem, a abóbora, a cenoura e a beterraba alimentos ricos em carboidratos não fibrosos, de fácil fermentação, esperava-se que o crescimento microbiano ocorresse de imediato, pois a velocidade de colonização é maior para alimentos com maior quantidade de carboidratos 
solúveis. Entretanto, existe alta especificidade inicial para a digestão do amido em função de sua origem (Cotta, 1988; McAllister et al., 1990). Dessa forma, se a dieta-padrão que é fornecida para o doador do inóculo não se aproxima daquela que foi incubada, é possível inferir que a necessidade de adaptação possa estar respondendo, ao menos em parte, pelo comportamento visto em relação ao período de duração da fase de latência. Mertens e Loften (1980) sugeriram que a presença do amido pode ter determinado o aumento na duração da fase de latência em estudos de cinética da digestão de alimentos in vitro.

Em relação ao parâmetro $m_{l}$, verificou-se variação significativa $(\mathrm{P}<0,05)$ em função dos diferentes alimentos estudados (Tab. 3). Em geral, houve maior taxa de degradação $m_{l}$ para a alfafa (fenada e peletizada), seguida da beterraba e da cenoura (Tab. 4). Essa maior taxa de degradação para as frações de carboidratos não fibrosos resultou em maior estimativa do rendimento microbiano sobre esses nutrientes (Tab. 5).

Tabela 4. Duração do evento de degradação inicial do alimento $(L)$ e taxas de degradação das frações de rápida $\left(m_{1}\right)$ e de lenta digestão $\left(m_{2}\right)$ de alimentos usados para várias espécies de ruminantes

\begin{tabular}{lcccccc}
\hline \multicolumn{9}{c}{ Parâmetro cinético } \\
\hline Alimento & \multicolumn{2}{c}{$L\left(\mathrm{~h}^{-1}\right)$} & \multicolumn{2}{c}{$m_{I}\left(\mathrm{~h}^{-1}\right)$} & \multicolumn{2}{c}{$m_{2}\left(\mathrm{~h}^{-1}\right)$} \\
\cline { 2 - 8 } & $\mathrm{N}$ & Média & $\mathrm{n}$ & Média & $\mathrm{n}$ & Média \\
\hline Batata doce & 28 & $4.04 \mathrm{a}$ & 28 & $0.136 \mathrm{c}$ & 28 & $0.027 \mathrm{~d}$ \\
Brócolis & 28 & $3.19 \mathrm{~b}$ & 28 & $0.145 \mathrm{c}$ & 28 & $0.032 \mathrm{~b}$ \\
Vagem & 28 & $2.98 \mathrm{bc}$ & 28 & $0.119 \mathrm{c}$ & 28 & $0.025 \mathrm{e}$ \\
Abóbora & 28 & $2.89 \mathrm{bc}$ & 28 & $0.154 \mathrm{c}$ & 28 & $0.029 \mathrm{c}$ \\
Feno de alfafa & 28 & $2.54 \mathrm{~cd}$ & 28 & $0.284 \mathrm{a}$ & 28 & $0.021 \mathrm{f}$ \\
Cenoura & 28 & $2.49 \mathrm{~cd}$ & 28 & $0.213 \mathrm{~b}$ & 28 & $0.032 \mathrm{~b}$ \\
Beterraba & 28 & $2.42 \mathrm{~cd}$ & 28 & $0.231 \mathrm{~b}$ & 28 & $0.036 \mathrm{a}$ \\
Alfafa peletizada & 28 & $2.07 \mathrm{~d}$ & 28 & $0.230 \mathrm{~b}$ & 28 & $0.022 \mathrm{f}$
\end{tabular}

Médias na coluna seguidas de letras distintas diferem entre si $(\mathrm{P}<0,05)$.

Tabela 5. Estimativas do rendimento microbiano (gcelulas/gcarboidrato) para a microbiota não fibrolítica $(Y c n f)$ e fibrolítica $(Y c f)$ da abóbora $(\mathrm{Ab})$, beterraba (Bet), batata-doce (B Doce), cenoura (Cen), brócolis (Br), alfafa peletizada (Alf $\mathrm{P}$ ), alfafa fenada (Alf $\mathrm{F}$ ) e vagem (Vag) para várias espécies de ruminantes

\begin{tabular}{|c|c|c|c|c|c|c|c|c|c|c|c|}
\hline Alimento-animal & $Y c n f$ & $Y c f$ & Alimento-animal & Ycnf & $Y c f$ & Alimento-animal & Ycnf & $Y c f$ & Alimento-animal & Ycnf & $Y c f$ \\
\hline $\mathrm{Ab}-\mathrm{boi}$ & 0,316 & 0,237 & Bet - boi & 0,304 & 0,269 & B.Doce - boi & 0,327 & 0,218 & Cen - boi & 0,298 & 0,240 \\
\hline $\mathrm{Ab}$ - búfalo & 0,305 & 0,222 & Bet - búfalo & 305 & 0,240 & B.Doce - búfalo & 0,290 & 0,195 & búfalo & 0,314 & 0,222 \\
\hline $\mathrm{Ab}$ - cabra & 0,341 & 0,230 & Bet - cabra & 337 & 0,269 & B.Doce - cabra & 0,260 & 0,210 & Cen - cabra & 0,321 & 0,240 \\
\hline $\mathrm{Ab}$ - ovino & 0,218 & 0,222 & Bet & 33 & 0,240 & ovino & 60 & 0,237 & $\mathrm{Cc}$ & 242 & 0,240 \\
\hline $\mathrm{Ab}$ - audade & 0,220 & 0,226 & Bet - audade & 323 & 0,282 & B.Doce - audade & 0,271 & 0,214 & audade & 0,317 & 0,264 \\
\hline $\mathrm{Ab}-\mathrm{c}$. sambar & 0,155 & 0,262 & Bet - c. sambar & 350 & 0,262 & B.Doce - c. sambar & 0,155 & 0,262 & Cen - c. sambar & 0,345 & 0,260 \\
\hline $\mathrm{Ab}$ & 0,271 & 0,257 & lande & & 0,230 & B.Doc & 0,277 & 0,257 & elande & 0,285 & 0,257 \\
\hline Média & 0,261 & 0,237 & Média & 306 & 0,256 & Média & 0,263 & 0,227 & Média & 0,303 & 0,246 \\
\hline EP & 0,066 & 0,017 & EP & 0,036 & 0,019 & EP & 0,053 & 0,025 & Ep & 0,032 & 0,015 \\
\hline limento-animal & $Y c n f$ & $Y c f$ & Alimento-animal & Ycnf & $Y c f$ & Alimento-animal & Ycnf & $Y c f$ & Alimento-animal & Ycnf & $Y c f$ \\
\hline $\mathrm{Br}$ & 0,301 & 0,264 & - boi & 325 & 0,252 & boi & 0,327 & 0,249 & $\mathrm{~V}$ & 0,287 & 0,255 \\
\hline $\mathrm{Br}$ - búfalo & 0,289 & 0,230 & Alf P - búfalo & 160 & 0,178 & oúfalo & 084 & 0,178 & búfalo & 0,186 & 0,233 \\
\hline $\mathrm{Br}-$ & 0,355 & 0,275 & Alf P - cabra & 0,324 & 0,237 & Alf F - cabra & 0,354 & 0,240 & cabra & 0,291 & 0,257 \\
\hline $\mathrm{Br}-$ & 0,222 & 0,240 & Alf $\mathrm{P}$ - ovino & 52 & 0,233 & Alf F - ovino & 0,334 & 0,218 & ovino & 0,333 & 0,237 \\
\hline $\mathrm{Br}-\mathrm{a}$ & 0,205 & 0,230 & Alf $\mathrm{P}$ - audade & 150 & 0,195 & Alf F - audade & 178 & 0,124 & Vag - audade & 0,130 & 0,067 \\
\hline $\mathrm{Br}$ - c. sambar & 0,155 & 0,262 & Alf P - c. sambar & 0,293 & 0,214 & Alf F - c. sambar & 0,348 & 0,218 & Vag - c. sambar & 0,267 & 0,246 \\
\hline $\mathrm{Br}$ - elande & 0,104 & 0,200 & Alf P - elande & 0,349 & 0,114 & Alf F - elande & 0,357 & 0,114 & Vag - elande & 0,100 & 0,124 \\
\hline Média & 0,233 & 0,243 & Média & 0,279 & 0,203 & Média & 0,283 & 0,192 & Média & 0,228 & 0,203 \\
\hline EP & 0,088 & 0,026 & EP & 0,087 & 0,047 & EP & 0,108 & 0,054 & EP & 0,089 & 0,076 \\
\hline
\end{tabular}

$\mathrm{EP}=$ erro-padrão da média. $\mathrm{C}$. sambar $=$ cervo sambar.

As estimativas do rendimento microbiano (Ycnf e $Y c f)$ foram maiores para a beterraba e para a cenoura (Tab. 5), o que, em princípio, é uma indicação para a utilização desses alimentos, especialmente, para os animais seletivos mantidos em cativeiro. A alfafa peletizada, a vagem e a alfafa fenada resultaram nos menores rendimentos microbianos sobre a fração dos carboidratos fibrosos. Talvez a maior concentração de lignina na parede celular desses alimentos possa explicar esse menor rendimento microbiano (Tab. 1). 
Ainda que as frações dos alimentos possam ser caracterizadas como de rápida ou de lenta digestão, diferenças de natureza química poderiam ser responsabilizadas por diferentes graus de utilização dessas frações pelos microrganismos presentes nos diferentes inóculos ruminais. Isso explica, em parte, o comportamento observado neste trabalho com relação à fração $m_{l}$, em que o inóculo de cada animal resultou em diferentes estimativas dos parâmetros frente aos diferentes substratos disponibilizados (Tab. 3 e 4).

As taxas de degradação das frações de digestão lenta $\left(m_{2}\right)$ mostraram-se sensíveis $(\mathrm{P}<0,05)$ ao alimento estudado, com valores significativamente maiores para os alimentos mais ricos em carboidratos não fibrosos, em comparação com os de menor concentração nesses componentes (Tab. 4). Esse comportamento reflete a natureza heterogênea da fração de carboidratos quanto à sua biodisponibilidade para a microbiota presente no inóculo.

De maneira geral, houve complementariedade das taxas fracionais, ou seja, nas situações em que a taxa de degradação dos carboidratos não fibrosos foi menor, observou-se maior taxa de degradação dos carboidratos fibrosos e viceversa (Tab. 4). Nessa relação, é clara a tendência de maiores valores de $m_{l}$ para os alimentos mais ricos em carboidratos não fibrosos e, por outro lado, maiores valores de $m_{2}$ para os alimentos com maior concentração de carboidratos fibrosos. Esse comportamento é esperado, uma vez que a dieta é o principal fator determinante da quantidade e dos tipos de microrganismos e, conseqüentemente, da taxa e da extensão da digestão dos nutrientes.

\section{CONCLUSÕES}

O modelo de duplo compartimento foi mais adequado para avaliar a duração do evento de degradação inicial do alimento e as taxas de digestão de diferentes tipos de alimentos pelas diferentes espécies de ruminantes. Não foram detectadas variações consistentes nas estimativas dos parâmetros cinéticos que permitissem agrupar os alimentos (fibrosos x não fibrosos) e os animais (domésticos $\mathrm{x}$ silvestres). $\mathrm{O}$ rendimento microbiano sobre os carboidratos (fibrosos e não fibrosos) foi maior para a beterraba e para a cenoura.

\section{REFERÊNCIAS BIBLIOGRÁFICAS}

COTTA, M.A. Amylolytic activity of selected species of ruminal bacteria. Appl. Environ. Microbiol., v.54, p.772776, 1988.

MALAFAIA, P.A.M.; VALADARES FILHO, S.C.; VIEIRA, R.A.M. Kinetic parameters of ruminal degradation estimated with a non-automated system to measure gas production. Livest. Prod. Sci., v.58, p.65-73, 1999.

McALLISTER, T.A.; CHENG, K.J.; RODE, L.M. et al. Digestion of barley, maize, and wheat by selected species of ruminal bacteria. Appl. Environ. Microbiol., v.56, p.3146-3153, 1990.

MERTENS, D.R.; LOFTEN, J.R. The effects of starch on forage fiber digestion kinetics in vitro. J. Dairy Sci., v.63, p.1437, 1980.

OFFICIAL methods of analysis. 15. ed. Arlington: AOAC, 1990. v.1. 117p.

RUSSELL, J.B.; O'CONNOR, J.D.; FOX, D.G. et al. A Net Carbohydrate and Protein System for Evaluating Cattle Diets: I. Ruminal fermentation. J. Anim. Sci., v.70, p.3551-3561, 1992.

SAMPAIO, I.B.M. Estatistica aplicada à experimentação animal. Belo Horizonte: Fundação de Ensino e Pesquisa em Medicina Veterinária e Zootecnia, 1998. 221p.

SCHOFIELD, P.; PITT, R.E.; PELL, A.N. Kinetics of fiber digestion from In Vitro gas production. J. Anim. Sci., v.72, p.2980-2991, 1994.

SICK, H. Ornitologia brasileira. Rio de Janeiro: Nova Fronteira, 1997. 912p.

SISTEMA de análises estatísticas e genéticas - SAEG. Viçosa:UFV, 1995.

VAN MILGEN, J.; MURPHY, M.R.; BERGER, L.L. A compartimental model to analyze ruminal digestion. $J$. Dairy Sci., v.74, p.2515-2529, 1991.

VAN SOEST, P.J. Nutritional ecology of the ruminants. 2.ed. New York: Cornell University Press, 1994. 476p. 